\title{
A planning and management infrastructure for large, complex, distributed projects-beyond ERP and SCM
}

\author{
George L. Kovács ${ }^{\mathrm{a}, *}$, Paolo Paganelli ${ }^{\mathrm{b}, 1}$ \\ ${ }^{\mathrm{a} C}$ Computer and Automation Research Institute, 1111 Budapest, Kende U13-17, Hungary \\ ${ }^{\mathrm{b}}$ Gruppo Formula S.p.A., Via Matteotti 5, 40050 Villanova di Castenaso, Bologna, Italy
}

\begin{abstract}
Enterprises which are distributed in space and/or which are composed as a temporary joint venture of legally different units recently often called virtual (extended) enterprises. Planning, design and operation (management) goals and requirements of such firms are generally different from those of single, centralized enterprises. The basic feature of an extended (virtual) enterprise is that the co-operating units of it keep their independence during the life-cycle of the co-operation-what is well regulated by the rules of the given conglomerate. It has to be accepted — on the other hand - that several basic functionalities and goals are the same for all types of distributed, large, complex organizations, which are the targets of our recent study.

The evolution of web-based manufacturing design/planning and operation system philosophies can be followed through the works presented in this paper. We intend to give software solutions for design, planning and operation (management) of complex, networked organizations represented as nodes of networks. In the first part of the paper, solutions are given to manage complex logistics flows of distributed SMEs, giving more sophisticated solutions than the commonly used supply-chain management (SCM) packages available in the market. The second problem we solve is a complex, web-based solution to manage large, expensive, multi-site, multi-company projects using any type of Enterprise Resource Planning (ERP) and flow management solutions. Our goal is to integrate as many available solutions as possible and to make only the appropriate frameworks including decision-support systems where necessary. The first part of the work means the establishment and application of a web server at each node of the co-operating network, while the second approach uses only one, joint web server and each node communicates with it through the network. These architectures are easy to be integrated if needed, i.e. logistic flows and project management can be solved together.
\end{abstract}

(C) 2003 Elsevier Science B.V. All rights reserved.

Keywords: Extended/virtual enterprise; Supply chain; Enterprise resource planning; WWW; Management; SME

\section{Introduction}

The traditional concept of business is obsolete. Companies, both manufacturing and service, are crea-

\footnotetext{
* Corresponding author. Tel.: +36-1-279-6140; fax: +36-1-466-7503.

E-mail addresses: gyorgy.kovacs@sztaki.hu (G.L. Kovács), paolo.paganelli@fromula.it (P. Paganelli).

${ }^{1}$ Tel: +39-51-600-2111; fax: +39-51-600-2222.
}

tors of value, not simply makers of products. Supplychain management (SCM) focuses on globalization and information management tools, which integrate procurement, operations, and logistics from raw materials to customer satisfaction. Future managers are prepared to add product value, increase quality, reduce costs, and increase profits by addressing the needs and performance of several things, such as supplier relations, supplier selection, purchasing negotiations, operations, transportation, inventory, warehousing, 
benchmarking, third-party vendors, electronic commerce, recycling, supply-chain electronic software, customer relations, etc.

Globalization underscored the need for supplychain professionals who seek a variety of experiences, who are committed to life-long learning, and who want to capitalize on ever-changing technology. Executive career paths increasingly are being recharted to mandate exposure to supply-chain functions. Supply-chain professionals are part of multinational and multi-functional teams and enjoy career opportunities of great breadth and depth.

Supply-chain management is one of the leading business process re-engineering, cost-saving and revenue enhancement strategies in use today. Effective integration of a supply chain can save millions, simultaneously improving customer service and reducing inventories; even greater gains are possible by bringing supply-chain strategies to the table early enough in the product development and design plans.

There are some SCM solutions available in the recent market. Naturally, they are all different, and only some, well-defined tasks can be solved by them, others cannot. To be able to select the best from different possible solutions, a deep analysis and appropriate simulation give a strong assistance. If it is possible to make experiments on possible scenarios it is easier to evaluate several "what-if" cases to find a good, or the best one. This way simulation gives assistance not only in technical, but in managerial decisions, too. Most available SCM solutions are for single, centralized organizations and they deal with restricted tasks, i.e. with management of the supply chain itself.

Today, the world-wide globalization and the appearance of virtual require more than only SCM for some tasks of a given enterprise. Due to the physically and logically distributed character of the co-operating units (workshops, plants, enterprises, etc.), taking advantage of the existence of Internet (intranet, extranet, etc.), web-based solutions are suggested.

These type of solutions are targeted by different projects world-wide. There are no plans for really general solutions, and it is hard even to imagine them. As far as the authors are concerned, there are two EU projects (FLUENT and WHALES, see [1,2]) to provide the "best" solutions. [1] gives "beyond SCM" workflow/supply-chain solutions for distributed (mainly SME) organizations dealing with manufacturing, services, maintenance, etc. The main target firms of [2] are the distributed, multi-site, multi-firm, powerful organizations (and SMEs), and the goal is to manage complex, one-of-a-kind products and projects, manufacturing and management as well.

In the following some solutions, innovative features and further details of the efforts and results of the complex flow control [1] and of the management [2] system will be given, taking into account that the two set of software solutions will be connected for applications if needed. Sections 2-4 are devoted to flow control, Sections 5-8 deal with project management issues, while some conclusions are found in Section 9 and there is an acknowledgement section to conclude. The solutions are developed not to substitute, but to be integrated with SCM and with the broader sense Enterprise Resource Planning (ERP) solutions. Such ERP candidates are, for example, Baan, SAP, etc. We summarize the basic requirements/advantages of webbased, "beyond SCM and ERP" solutions at the beginning of Section 2 (flow management) and Section 5 (project management).

\section{Management of complex logistic flows}

The results of our R\&D efforts provide new IT solutions for managing complex logistic flows, occurring in distributed manufacturing networks with multiple plants and co-operating firms. Networks of this kind are gaining relevance and diffusion, under the impulse of the following main factors:

- emerging virtual/extended enterprise paradigms;

- pull-oriented production models, like just-in-time, requiring synchronization of internal and external flows;

- lean/agile manufacturing models, based on horizontal, goal-oriented process chains;

- evolving market conditions, calling for business globalization and decentralization of manufacturing facilities.

In response to these changes, the newly developed solution and software provide manufacturing firms with advanced IT tools for logistics decision-making, thus enhancing their capability to operate in a distributed production environment. 
Our objective is to enhance the firms' capability to operate in a distributed manufacturing network. This kind of organization has been attracting great interest from the industrial community world-wide, under the impulse of innovative paradigms and evolving market conditions.

- Much attention is presently paid to the concepts of virtual/extended enterprise, intended as a network of autonomous firms that co-operate in achieving common business goals. When applied to manufacturing, these models imply changes and extensions to the firms' interaction with the external world, with a consequent strong impact on the context and scope for logistics decision-making [7].

- Advanced "pull" techniques, like Kanban, emphasize the importance of smoothing and synchronization of production tasks at the shop-floor level. A critical success factor is to reach equivalent timeliness and coordination with processes outside the factory, thus calling for increased commitment and reliability of both supply management and the suppliers themselves.

- Lean/agile manufacturing models propose the shift to a more process-oriented enterprise structure, where value-adding functions are highlighted and involved into transient, goal-driven process chains. To succeed, this approach requires the same flexibility in the management of business partners, and improved control along the entire supply chain [8].

- Even in a traditional organization where such innovative concepts are neither applied or envisaged, the complexity of logistics decision-making is now increased by factors such as: market globalization, decentralized manufacturing facilities, extended range of suppliers, highest emphasis on total quality issues and customer satisfaction $[9,10]$.

In these conditions, traditional logistics functions like sales and purchase are left alone to face problems far beyond their intended roles. Current Enterprise Resource Planning systems can be of little help, only supporting the administration of conventional customer-supplier relationships with basic services like ordering, invoicing and inventory. These systems are clearly insufficient to cope with the new manufacturing scenario, based on decentralized and flat organization models in conflict with the ERP foundations of:
- hierarchic organization, with strict planning rules and pre-determined chain of responsibility; this is in most cases an obstacle to include external actors, like sub-contractors or partners into the decisional process;

- embedding of business processes into the application code; this makes it impossible to manage complex logistics flows, involving business partners, unless these are simulated by, e.g. "special" routings and work-centers.

- centralized data management based on companyspecific standards; this prevents full integration with other firms' information systems, thus limiting information sharing and co-operation in the distributed organization.

Problems deriving from these evident limitations often induce large companies to assimilate their closest suppliers and sub-contractors, at least from the information system point of view. On the one side, this solution can find resistance from the involved partners and, in most cases, it results in makeshift systems with very low flexibility. On the other side, for the partners it means strong renounces in terms of decisional autonomy and, if they are SMEs, the additional costs of an over-sized ERP system to comply with their big customer standards.

To overcome these limitations, major ERP producers are developing supply-chain management addons on top of their production management solutions, often through partnerships with SCP producers. Great diffusion of these systems is foreseen in the future, as testified by the activation of large supply-chain planning projects in leading industries world-wide. In spite of their technological content, current SCP systems are still bound to state-of-the-art ERP software, with consequent significant limitations.

- Centralised planning: as ERP add-ons, SCP systems simply extend traditional MPS and MRP planning with new functionalities, such as distribution and transportation planning, and improved performance, thanks to memory-resident algorithms. Planning is still performed from a static, centralized view of the supply network, with neither real-time integration nor negotiation with the actual nodes.

- Manufacturing versus logistics orientation: even though an attempt is made at managing the complexity of enterprise networks, this is still done from 
a manufacturing perspective, i.e. applying hierarchic planning approaches where external firms are assimilated to internal shop-floor isles or cells.

- Pre-defined organization model: the supply-chain organization and accountability structure is coded into the application software, and hence can be hardly adapted to the different and mixed partnership models found in the real world.

- High implementation costs: due to the complexity of SCP integration with the existing organization and information system. Ad hoc links must be developed for each data source, either inside or outside the enterprise, in order to feed the SCP static models. This poses technical problems of data reliability and consistency, and still greater organizational problems of data ownership and maintenance responsibility.

In spite of the increasing market interest, originated by the actual end-users difficulties and by the ERP functional deficiencies, SCP solutions still appear as sophisticated planning engines for isolated, highpower decision makers. Tactical decisions and dayto-day coordination between trading partners are still based on informal communication or point-to-point integration realized, e.g. through batch EDI transfers.

\section{A novel supply network/flow control model}

Traditional SCM implementations refer to a linear, standardized and relatively stable view of the supply chain: "Supply chain management is about managing the flow of products and services and the associated information, across the whole business system to maximize value to the end consumer" [11].

The interpretation given by SCP vendors to this definition is often reductive. The "whole business system" is a row of four to five actors (depending on whether electronic commerce issues are addressed or not) interacting with each other in pairs (Fig. 1).

The resulting SCM solutions are product suites including several independent tools, each designed to optimize a single link in this pre-defined sequence. For the whole picture to work, it is assumed that separate optimization of each link leads to overall performance improvements.

Recent analyses have pointed out the potential failure behind this logic, especially where revenue increase is pursued instead of cost reduction.

- Cost reduction leads to standardization and simplification of supply chain and its operation; minimization of integration costs; definition of "functional silos" independent of each other.

- Increasing revenues means to take advantage of diversification and differentiation, exploiting changes in demand and supply. This means making more money thanks to the supply-chain ability to reconfigure itself, to harmonize capacities and to respond quickly as a whole.

To look at the supply-chain complexity as a competitive advantage, rather than as a source of costs, means a radical change of perspective in the organization models supported by SCM tools: "For a start, the supply "chain" is really not a chain at all-it is a complicated web of relationships between demand and supply. The concurrent and multidimensional

\section{Information flows}

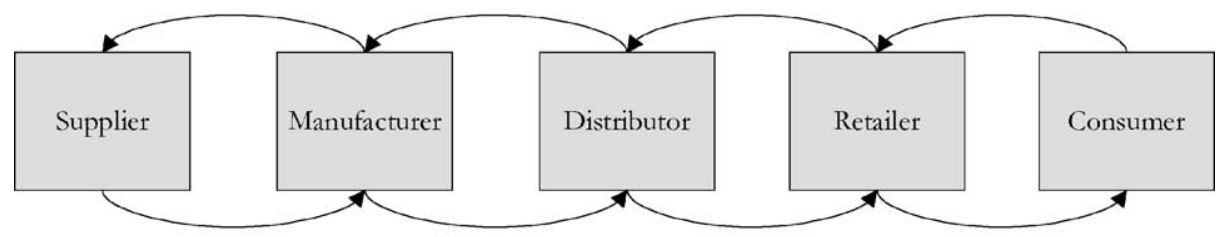

Materials flows

Fig. 1. Traditional supply-chain representation. 

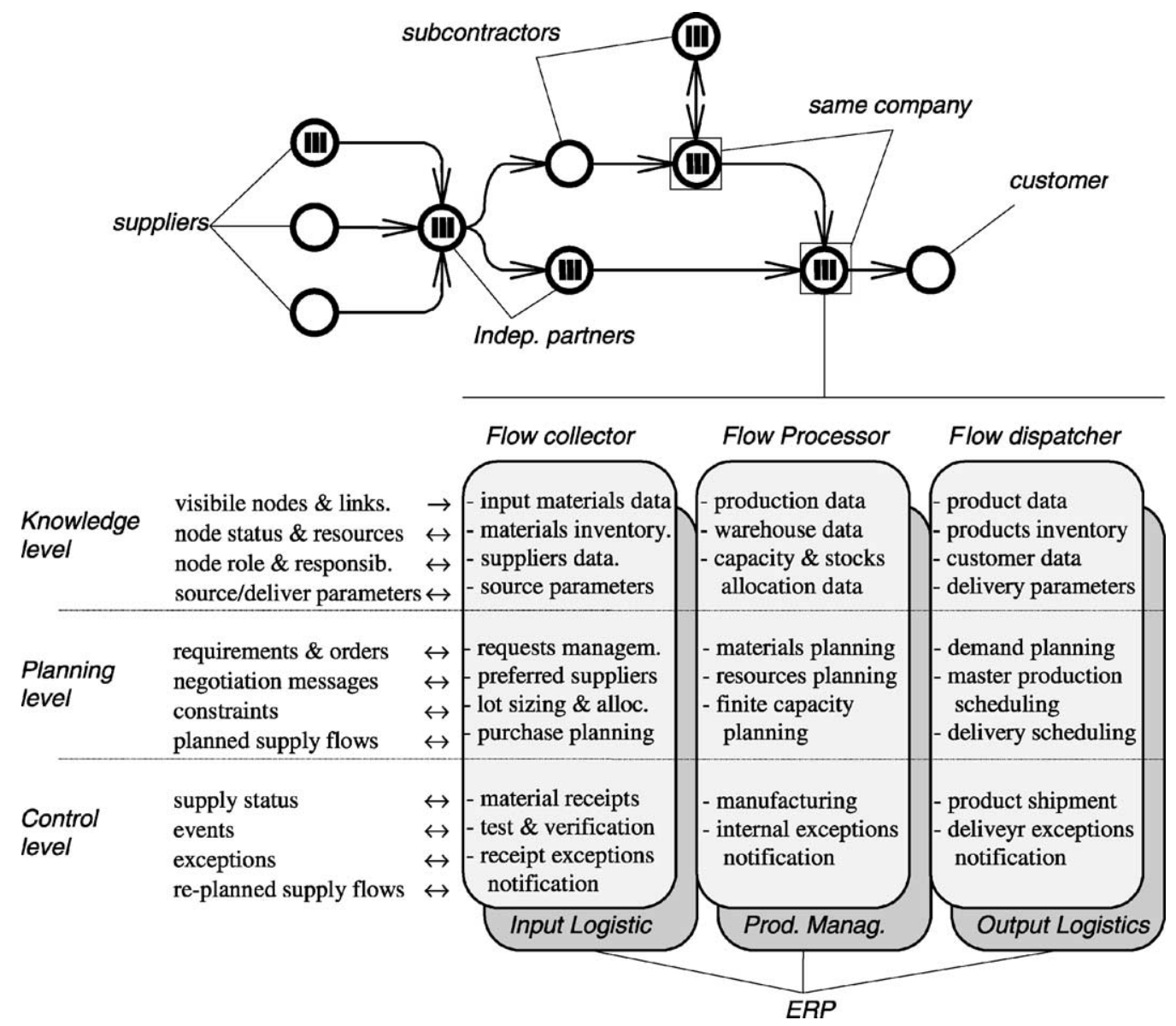

Fig. 2. The novel supply network model.

nature of these relationships creates a complex fabric woven step by step" [12].

Our solutions capture the inherent complexity of the supply network, allowing firms to manage the three fundamental barriers to supply-chain performance improvement: visibility, velocity and variability. This is done by referring to a flexible, scalable and decentralized network model, based on the preservation of nodes autonomy and on a case-by-case definition of links and dependencies between the nodes.

The logical architecture of our network is given in Fig. 2, where circles including three bars represent nodes equipped with the new system, and empty circles represent other nodes acting as customers, suppliers or sub-contractors. Nodes of the latter type can only take part as executors in logistic flows controlled by the flow management nodes. The reason is that these nodes lack the network-level vision and decision-support tools to actively participate in the planning and coordination of supply flows.

Each node is perceived by the other nodes as an autonomous source of: (i) information on the node and the goods it supplies and consumes (knowledge level); (ii) demand/availability signals and allocation decisions (planning level); (iii) supply control signals and exceptions (control level). Independent of ownership and position in holding hierarchies, nodes in the network are modeled as source and destination of logistics flows. For this purpose, each node is attributed a three-tiered structure including: a Flow Collector, that manages incoming logistics flows, a Flow Dispatcher, that manages outgoing flows, and a Flow Processor, 
responsible for integration with internal production flows.

Co-operation between nodes is realized through links, each representing a stable relationship for the exchange of a given product between a "supplier" node Flow Collector and a "receiver" node Flow Dispatcher. The Flow Processor is not directly involved in the link, since our flow control is based on a clear separation of logistics decision-making domains. Internal logistics are managed by each node on its own, and are perceived at the network-level only through requirements, events and constraints on external logistics flows.

A link definition fixes the characteristics of supply flows taking place through the link, in terms of:

- data on the supplied product, including shipping, transportation and delivery parameters;

- planning policy applied to the link, in terms of planning parameters, planning method, e.g. "push" or "pull", and planning responsibility, e.g. either the supplier or the receiver, or a third node controlling the flow;

- workflow model, i.e. the sequence of messages and events characterizing the nodes interaction during planning and control of supplies over the link.

In this way, a high degree of generality and flexibility is reached in modeling the variegated network configurations found in the real world. For example, a node can establish "pull" links with a network of suppliers, keeping a centralized control of suppliers selection and orders allocation. The node product can be delivered to a trading partner on the basis of an inventory replenishment agreement, modeled by a "push" flow controlled by the supplier, and to a customer on the basis of a normal "pull" link. Both types of outgoing flows can originate dependent requirements for the above suppliers network.

\section{System software requirements and possibilities}

To support the above detailed organization model, each node is provided with innovative software tools designed to fulfil the requirements of a multi-site, multi-enterprise manufacturing network. First we show the software requirements specification and then some solutions are given.

\subsection{Software requirements}

There are some basic requirements along which the software was designed and implemented. These have the following main goals.

- To provide a unified and generalized representation of logistics flows at a proper detail level; this means linking supply and demand sources across the network, but masking local production activities within each node.

- To support decentralized and volatile organization models; to be general and commercially exploitable, the system does not rely on any pre-defined network schema, but supports a case-by-case definition of nodes relationships, from external supply to sub-contracting, to co-operation in a virtual enterprise.

- To allow scalable and flexible network configuration; the system is open to inclusion of new nodes, and supports the individual node in readjusting its role and interface toward the network for, e.g. reacting to changes in the network organization, or making new resources available to co-operative manufacturing.

- To support decision-making at the tactical and operational level; this means, for example, to select potential partners on the basis of their past performance and capabilities, to plan the materials and activities flows triggered by an incoming order, and to substitute a failing or delaying node in a running flow.

- To manage and synchronize multiple decisional processes; the system supports distributed decision-making schemes, assigning responsibilities to each node according to the role played in the manufacturing network, resolving potential conflicts, and integrating multiple decision threads in a consistent and transparent fashion.

- To integrate and distribute relevant information across the network; supply-related data maintained by each node is harmonized and integrated into a generalized data model, and it is made available to the other nodes according to visibility rules mirroring the network organization model and decisionmaking scheme.

- To integrate but not overlap with ERP and other internal-logistics management tools. The system 
should not interfere with a node production planning mechanism, this representing a confinement of the node autonomy and hence a limitation to the network survival and extension; instead, proper integration is provided with local logistics management functions.

To avoid replication of functionalities and waste of resources, we strongly rely on the integration with standard tools. In particular, our communication and workflow infrastructure is based on commercial tools for data interchange, messaging and process automation, compliant with standard network protocols and widespread operating systems.

\subsection{Software capabilities and components}

To fulfil all goals and requirements, we provide an advanced IT infrastructure based on the following software components (see Fig. 3).

- A standard communication and workflow infrastructure, for basic data interchange and message services. It can be easily accessed and configured to realize higher level functionalities.
- A high-level network model, constructed on top of this basic layer to: (i) provide and update a consistent representation of the network from the node point of view; (ii) describe the network physical layout and accountability structure; and (iii) adjust the node view and decisional power to network-level changes.

- An active flows control (AFC) component, which monitors interaction with nodes in the network model to: (i) maintain updated information on active logistics flows; (ii) dispatch relevant messages and events from and to the decisions-support components; and (iii) support negotiation with the other nodes.

- A performance measurement system (PMS), acting in parallel with the AFC to: (i) keep historical recordings of the network activity; (ii) calculate significant performance indicators; and (iii) provide multiple performance measures for the different network roles and viewpoints.

- Two decision-support systems (DSS), respectively for input and output flows management, that: (i) process internal and external demands; (ii) allow flow planning based on AFC and PMS input, according to the node role and policy; and (iii) react to

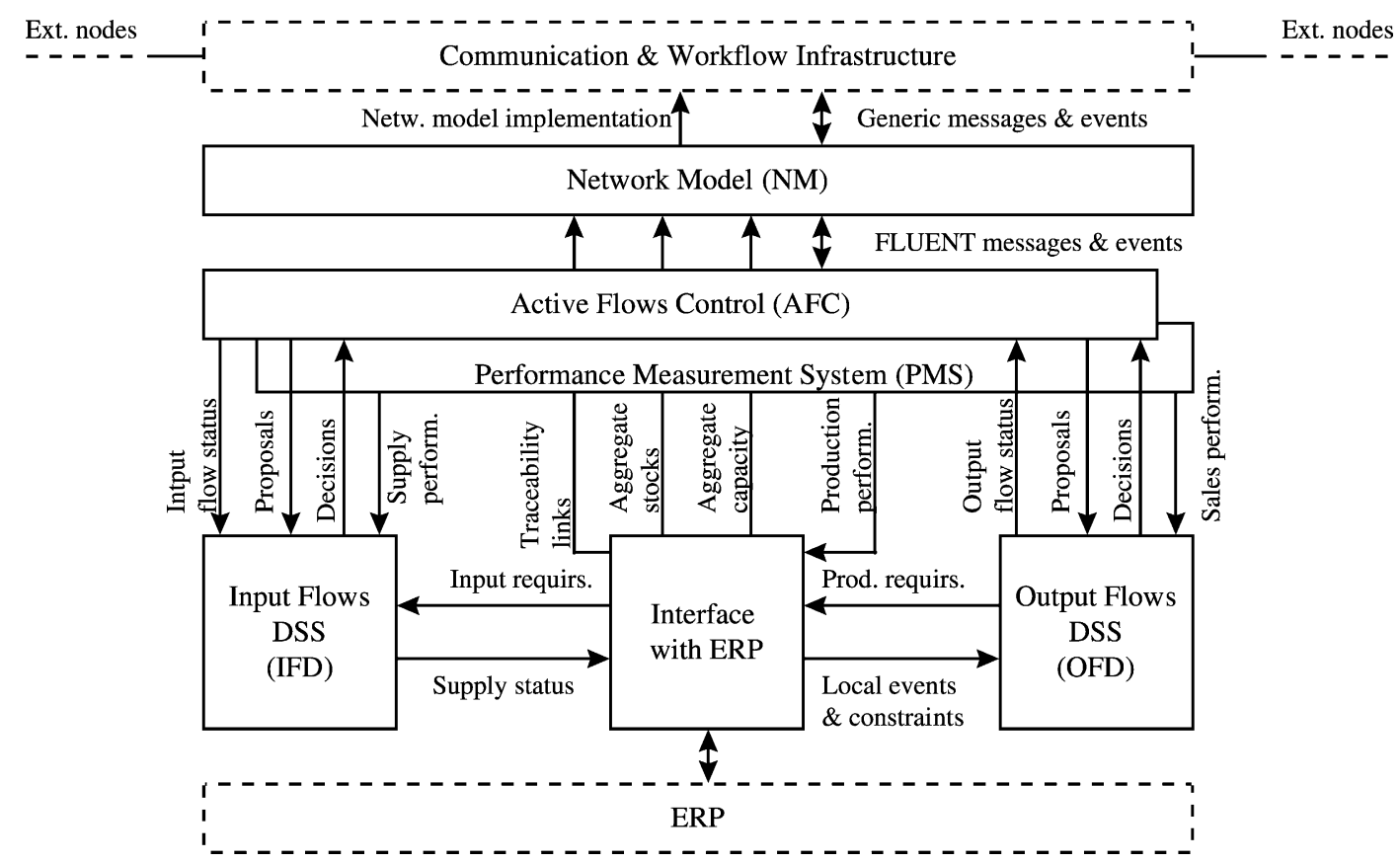

Fig. 3. Workflow node IT architecture. 
exceptions and negotiation messages from other nodes.

- An interface with enterprise resource planning that allows transparent interaction with the node local production management, in order to: (i) supply aggregate capacity and stocks information to the network model; (ii) exchange requirements and status information with the DSS; and (iii) maintain traceability between incoming and outgoing flows.

\section{Project management issues}

\subsection{Supporting integrated planning, deployment and monitoring of large engineering projects}

The objective of our work in this chapter is to provide a planning and management infrastructure for complex, distributed, multi-site, multi-enterprise organizations working on large-scale engineering projects, characterized by huge investments in both materials and human resources (HR) and by concurrent, disparate activities (manufacturing, design and services) as well. Managing projects of this kind means dealing with several problems at the same time.

- Complexity of scope, in terms of time and resources employed, and variety of activities to be planned, synchronized and monitored.

- Distributed organization, spanning through several companies and involving a multiplicity of actors and competencies.

- One-of-a-kind design, increasing planning complexity, hard to apply product and process standardization.

- Geographic distribution of project activities, sometimes in unprepared or hostile environments.

- Strict time constraints, with complex milestones and dangerous critical-path dependencies.

- Contingency risks, due to the high planning uncertainty and difficult re-alignment of activities.

- Revenue-loss risks, due to difficulties in budgeting and high contingency costs.

Concerning the research state-of-the-art, we can identify two main directions pursued in the last years by many projects.

- On one side, standards and systems are sought for product and process data modeling and interchange, and to support distributed design in concurrent and co-operative engineering environments. This category of projects focuses on the "what", i.e. on the contents specifications for a product or project, rather than on the "how" and "when" that are typical project management concerns. References to some of these projects are in $[3,4]$.

- On the other side, virtual enterprises are studied as evolving organisms, investigating environmental, legal and socio-economic conditions for the creation of enterprise networks. Considerably, less effort has been directed to the analysis of the planning and monitoring problems characterizing such networks, and to how co-operation can be sustained and managed on a daily basis. References to some of these projects are in $[5,6]$.

Both categories of projects follow a different approach from the one we follow, and their results are to a large extent complementary to our system. The innovation of our system lies in its focus on the planning and day-to-day management of large projects, assuming that technical departments and engineering functions have proper working tools and standards, and that conditions for virtual enterprise creation have been fulfilled in the specific cases involved. This is probably true, if the organization is actually planning or managing a project).

These problems are important for most users, who are actually lacking any ICT support in dealing with complex distributed projects' management. The proof is in the fact that many firms operating on such large-scale projects, have developed, or are in the course of developing "in house" project databases and project management tools. These systems lack the distributed features and model sophistication of our system, but are nevertheless a partial answer to problems currently not addressed by systems in commerce and by research and development projects we are aware of.

In response to these requirements, our system pursued two main objectives:

1. to design and develop a set of software components supporting integrated planning, deployment and monitoring of large projects in multi-site, multi-enterprise organizations;

2. to demonstrate the applicability and benefits of the developed software components through different 
pilot business cases presented by analysis, implementation and experimental usage.

As an innovative system for project management in complex and distributed organizations, the system implements the following general features.

- Provides a unified and generalized representation of project activities and related artifacts, comprising all material and immaterial work items (e.g. products, knowledge, design documents in different stages) that need to be organized in complex projects. Supports distributed organization models, crossing hierarchies and company boundaries; to be general and commercially exploitable, the system does not rely on any pre-defined organization schema, but supports a case-by-case definition of links between companies, organizational units and employees involved in each project.

- Provides a scalable and flexible co-operation environment. The system provides a project network infrastructure accessible to every node (company or organization unit) independently of its size and information system. It supports nodes and individuals in readjusting their role and interface toward the network (for example, to reflect changes in the node internal organization, or to make new resources available to any project).

- Integrates and distributes relevant information across the project network. Data maintained by each node and related to a specific project is given a generalized representation and shared with the other project participants through a web-based environment according to visibility and consistency rules mirroring the project organization model and management responsibilities.

- Supports decision-making in the project ideation, definition and deployment phases. This means to select potential partners on the basis of their past performance, cost and capabilities, to generate detailed plans considering both activities' timing, equipment and materials availability, and to find substitute resources for a running activity, etc.

- Manages and synchronizes the flow of decisions and events in the project network. The system manages the distributed workflow associated to a project, e.g. circulating planning proposals among the partners, integrating multiple decision threads in a consistent and transparent fashion, and dispatching monitored exceptions to the responsible actor(s) for contingency management.

- Integrates with local management and planning systems. It means to safeguard the nodes' autonomy and IT investments. The system shall not interfere with node internal procedures and management tools, as ERP, PPC, human resources, stand-alone project planning and budgeting packages. Instead, proper interfaces are designed and implemented for real-time information exchange between these systems and our new management system network infrastructure.

Such projects are rarely carried out within the scope of a single organization. More often, the prime contractor, typically a large company with adequate know-how, references and financial resources to sustain the project, outsources specific components and services to smaller firms through several forms of subcontracting. This way, SMEs are often involved.

The previously listed general features answer the following problems, too:

- high direct and indirect costs of basic resources;

- complex and hierarchical organizations grown up in times of unchallenged and stable demands (e.g. markets protected by local governments);

- low operative margins, putting short-term activities and contingency management ahead of technology and business process improvements;

- cultural and organizational obstacles to apply "virtual enterprise" partnership models;

- low flexibility that, paired with complexity, makes it almost impossible to prepare reliable plans and project budgets.

As stated earlier, the prime contractors in largescale projects are typically big companies with proper financial resources and assets. Nevertheless, this does not prevent our system to be extremely significant to SME users that can be involved as nodes (e.g. subcontractors for provision of services and components, to develop entire engineering packages, etc.) in a large project network.

\subsection{The main advantages and their measurement for project management}

A good project control/management software assists in project planning and deployment thanks to 
a software infrastructure producing the following measurable results on the end-users' business:

\subsubsection{Improved planning and budgeting}

These are measured in percentage of successful bids, and in planned versus actual costs/duration, etc., and are achieved by means of designing and implementing several subsystems, and taking into consideration several important factors at the same time.

- A planning and financial analysis system for bid preparation (tendering): projects managed by our system have to go over a hard preliminary competition, commonly called "tendering". The price, the deadlines, the technical specifications have to be given in a bid, so a poorly calculated bid can result winning the order but failing the profit and the delivery terms.

- Considering the whole scope of project activities (including sub-contracted ones, as well as needed materials, machinery and human resources at all project sites) when taking project timing and allocation decisions.

- All decisions are based on updated and consistent information about running activities and availability of resources, on aggregating and normalizing data from heterogeneous applications and different functional domains both inside the company and across any given participating network-using appropriate DSS.

- Analyzing and comparing alternative scenarios, generated through alternatives on a full-scale project model, and evaluated and compared by means of advanced on-line analytical processing toolsusing appropriate simulation and DSS tools.

\subsubsection{Improved monitoring, cost and risk assessment}

These are measured in manpower/assets utilization, in reduction of "idle" time (waiting for unfinished activities), etc. and they are achieved by means of:

- on-line access to the current status of project activities (the resources consumed at all project sites, the quantifiable results and costs borne, and any other indicators relevant to project progress evaluation and current risk assessment);

- real-time notification of events and conditions constituting potential failure sources to the appropriate actors in the project network, according to rules and criteria set case-by-case (e.g. maximum delay on a critical path activity);

- automatic update of the project plan, highlighting deviations between actual and planned activities, their impact on related tasks and milestones, and corrections required to meet the project objectives.

\subsubsection{Effective contingency management}

This is measurable by the percentage of "perfect orders", i.e. orders delivered according to original request, and by the reduction in number of cancelled or re-negotiated orders, and are gained by:

- pro-active risk analysis in the project planning phase, where alternative solutions are compared considering both internal factors (e.g. strict time-dependencies) and external factors (e.g. casual distribution of machine failure or manpower shortage at project sites);

- re-planning in the project deployment phase in reaction to alerts and deviations notified by monitoring/DSS functions, selecting backup options on the basis of cost, perturbations on running activities, need for re-negotiation of already set plans, etc.;

- alignment of the whole project plan to changes on re-planned activities, promptly updating all project sites and management levels, and tracking of project revisions for costs evaluation and statistical risk analysis (DSS).

\subsubsection{Higher flexibility and efficiency}

The best measures for them are the increase in bids processed by the same organization, the reduction of bid preparation cycle time and the profit margins by order and project unit. These can be approached by:

- ability to respond quickly to customers' requests for proposals and requests for changes by involving the appropriate technical and management skills at all project sites;

- better exploitation of the network resources, thanks to a decision-support environment which is aware of co-operation possibilities (e.g. roles to be fulfilled in a project under planning) and available partners' skills and capacities;

- prompt negotiation of planning and re-planning options, by means of a communication infrastructure that circulates decisions and events between the appropriate actors, crossing companies and organizational units boundaries. 


\section{System software environment issues}

To achieve the above improvements requires dealing with different enterprise functions and information sources, supported by heterogeneous and poorly integrated software applications, as:

- Enterprise Resource Planning systems (as SAP, Baan, etc.) represent the companies' administration backbone, and provide basic transactions for bids and contracts management, job order stages and costs reporting, billing and invoicing;

- Production Planning and Control (PPC) and Warehousing systems, often sold as ERP components, support materials management and long- to shortterm production planning;

- project planning tools provide graphical editing of GANNT and PERT project diagrams, along with on-line display of resources workload and activities timing;

- human resources packages support company organization management, identifying key project roles, skills and positions, as well as project personnel costs and timetables.

None of these systems by itself covers the full spectrum of project management requirements, that in complex organizations range from financial planning and cost analysis, to human resources recruiting and assignment, to procurement and allocation of manufacturing resources and materials. Moreover, none of these systems provides a data and communication infrastructure for the whole project network, i.e. to the multi-site, multi-company organization created to fulfil specific project objectives. As a temporary and goal-oriented structure, although it can last years and absorb large turnover shares, the project network presents typical "virtual enterprise" properties that make it impossible to map it on traditional, enterprise-centric information systems.

\section{Some innovative features of the project management system}

The most innovative aspects of our system solution lie in its distributed architecture design, that provides an integrated data and process infrastructure for different companies and actors participating in large projects' planning and execution, at the same time safeguarding each node's autonomy as regards local operations management and information system. These features match key requirements of the so called "virtual enterprise" organizations working on large one-of-a-kind industrial projects, as it was recently highlighted by a survey on European largescale engineering companies carried out in the IV Framework EU Programme [13]. These are: lack of data models, communications and workflow infrastructures for project teams "extended" to suppliers and sub-contractors; lack of life-cycle planning, costing and risk assessment tools for complex distributed projects.

State-of-the-art software applications offer only partial responses to the above needs, being still too much dependent on given, specific industrial sectors, organization models or ERP platforms, and approaching project management with a solution-oriented rather than with a problem-oriented approach. They are focused on specific tools or technology applications to optimize a single aspect of project life-cycle management.

(1) ERP packages' Management extensions. ERP systems are being universally adopted as the enterprise backbone for execution functionalities, and world-class packages (e.g. SAP, JDEdwards, Baan) provide project management modules capable to integrate typical ERP functions like job orders management, accounting and purchase, with higher level features like Work Breakdown Structure (WBS) or project profitability analysis. These solutions are characterized by the low flexibility and the centralized management of the project model. The best fitting works that are centrally planned and executed, and not frequently revised.

(2) Project management applications include a wide range of stand-alone software products, such as: (i) professional project planning and project accounting suites (e.g. SAS, Solomon Software, etc.) provide advanced decisions support, scheduling and on-line analysis features; (ii) office project applications (e.g. MS Project) provide graph- and table-based editing for manually planned projects, easy to use and integrated with common office tools; and (iii) dedicated packages 
provide a broad range of project management features for specific industrial sectors (e.g. ABT for software development projects, others in many sectors).

(3) Data interchange and workflow infrastructures have recently emerged as means for improving efficiency and standardize operations of complex distributed organizations, including engineering networks. On the one side, standards for data and documents interchange (e.g. STEP, EDI) provide the foundations for knowledge sharing and communication of engineering and commercial information. On the other side, communication and workflow technologies provide process automation features for real-time electronic business interactions. Yet, technology alone fails to give users a full view of their project networks, and leaves unsolved many functional requirements that go beyond the simple exchange of data and messages.

Our system introduces a significant advance in project management practices supported by state-ofthe-art applications, thanks to a flexible architecture integrating project-related data from heterogeneous applications, workflow automation, and decision-support functions into a web-based environment.

The resulting system accommodates the needs of project networks independently of the industrial sector, thanks to its general and adaptable design, that comes from the following features.

\section{Distributed project management environment. The} system provides a unique entry point to all data and activities associated to nodes in the project network, and will allow independent data maintenance and interoperability to actors belonging to any node. The absence of clear distribution functions is the main problem of current project management solutions, contrasting with the geographically and functionally distributed project network organization. Only top-class ERPs support multi-site installations, yet, the enterprise-centric ERP model poses an "all or nothing" dilemma as regards integration with project partners: either they are modeled as subsidiaries (and buy the same system), or as external customers or suppliers. With our system, a third-party service provider, or the above example's remote site manager will be able to insert resources and activities accounting data from any location directly into the shared project repository.

2. Decentralized architecture and accountability structure. This will make integration between business partners easier, avoiding imposition to the nodes of an external control entity and centralized information system standards. The adoption of a centralized approach is one of the major obstacles for exploitation of virtual/extended enterprise models and supply-chain management tools. With the new system, each node will remain in charge of its own data and local applications, without giving up control of internal operations and without changing its information system architecture.

3. Powerful project and network data model, allowing for detailed representation and configuration of different project structures and network organizations. This is a key feature since, to be effectively applied in various industrial sectors, the system cannot rely on a rigid model mirroring the activities, resources and organization of a specific industry. This is the approach taken by vertical project management applications, trying to cover all project details in a specific domain but failing whenever real users' cases do not fit in their prepackaged models. On the other hand, general purpose project management packages and ERP provide very simplified models, failing to capture the variety of project activities and resources, and the complexity of their relations and constraints.

4. Project representation takes into account both materials and resources requirements, and maps them onto a model of the physical distributed project network, its various types of resource, and its accountability organization.

5. Flexible decision-support tools. The software components are designed to cope with decisionmaking requirements of the various actors involved in project management and monitoring activities, operating at different nodes and in different stages of the project life-cycle. Advance will be in terms of exploitation of the networklevel visibility and powerful data model, rather than in algorithms sophistication or graphical visualization features. Current packages already provide powerful scheduling, on-line analysis and 
reporting features. With our system, the decisionmakers gain visibility of different types of activities (e.g. engineering, manufacturing, procurement) and resources along the project network, with the possibility to examine alternative configurations and multiple constraints (e.g. resources time and materials availability) provided by the network data model.

It is worth observing that the decentralized and flexible project management model is able to safeguard the autonomy and visibility of each network node, independently of its size. This prevents the constitution of hierarchic project networks, actually dominated by a single, large contractor. Typically this happens when planning and logistics departments of large firms, faced with tasks surpassing their traditional responsibilities, tend to pass part of this complexity on to their suppliers. These are often SMEs, whose resources and commercial strength are insufficient to deal with such demanding scenarios, with consequent problems in terms of competitiveness losses and high risk on investments.

\section{The structure and relationships of the system components}

The system architecture has been designed to match the project wide application scope, the complexity of technical objectives, the variety and extent of business cases to be analyzed and implemented at pilot users' sites. Each of these topics raises different categories of problems, requiring specific competencies along with conventional project management and software development activities. For this reason, the work has been subdivided into two thematic areas (see Fig. 4).

The parallel and interactive design of these two areas was a basic idea to build up the software system where the user requirements, models and tools were developed in close connection to each other giving a fast feedback every time it was necessary. This way all the software components were designed and implemented taking into account real, industrial requests all the time.

The two basic thematic areas in the system are as follows.

1. Network architecture and software components: The project main body consists of five technical parts devoted to the study and development of the ICT architecture and software components that support the system network organization model. Each part included the fundamental activities of a quality-based software development process: requirements, analysis and design, implementation, test and deployment. A sixth part provided a common development infrastructure for the teams working on each component, dealing with: methodology and tools to be used, selection of existing

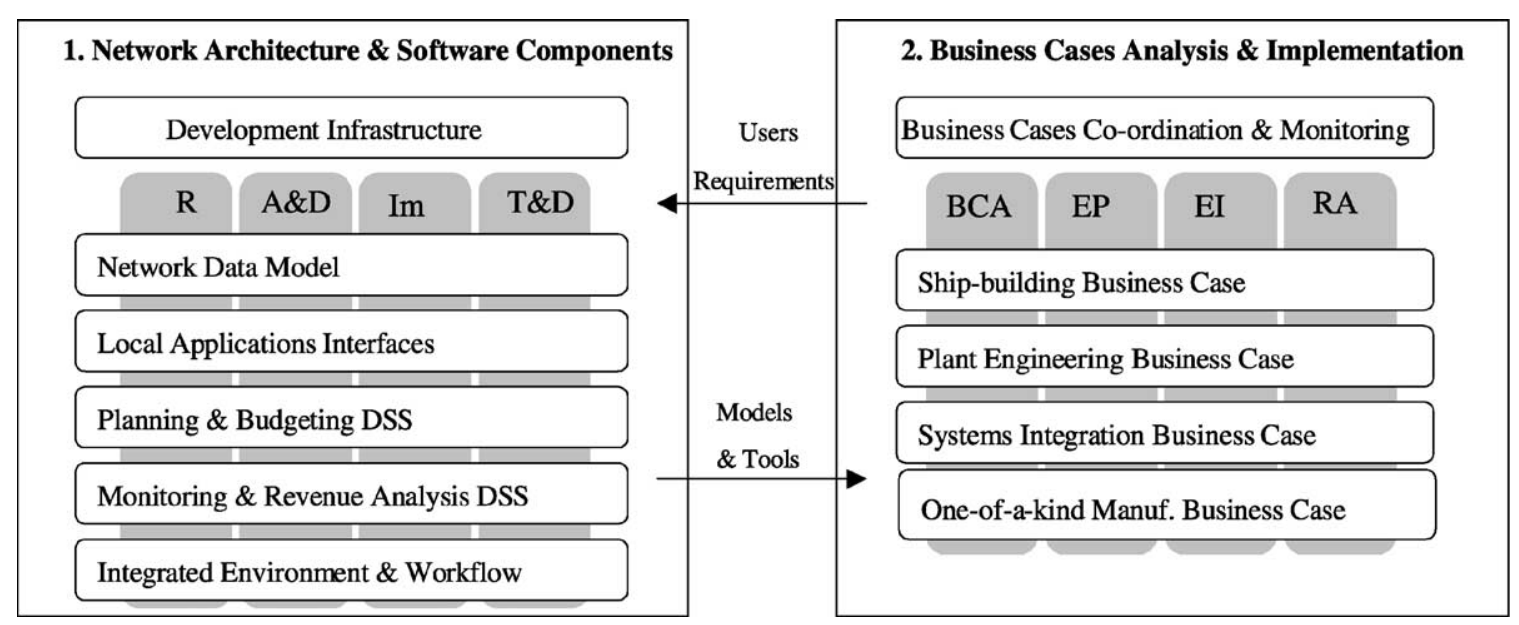

Fig. 4. Project management system architecture. R: requirements; A\&D: analysis and design; Im: implementation; T\&D: test and deployment; BCA: business cases analysis; EP: experiment preparation; EI: experiment implementation; RA: results' assessment. 
re-usable components, coordination of joint developments, maintenance of a technical data repository, configuration and change management.

2. Business cases analysis and implementation: In parallel with technical developments, the proposed organization model and tools were introduced and applied on four business cases proposed by users in different industrial sectors (see the right-hand side of Fig. 4). Each business case consists of the fundamental activities of business case analysis, experiment selection and preparation, experiment implementation and results assessment.

All business cases are coordinated dealing with application of common methodologies, metrics and best practices, and ensuring uniformity, comparison and joint evaluation of results produced by each business case.

Fig. 4. shows the main relationships between the thematic areas by arrows, as follows.

- User requirements produced in business cases analysis were necessary input to software analysis and design specifications.

- Model specifications and software tools produced in the technical packages were necessary for experiments preparation and implementation of all business cases.

As it can be seen in Fig. 4, the results of our system are demonstrated by four different pilot users with different pilot cases, in different countries.

- Lisnave, an important Portuguese company with a long tradition in shipbuilding and ship-repairing services, is presently suffering aggressive competition from the far East.

- FATA, large engineering company of north-west Italy, represents a complex and hard-to-manage business with respect to leaner and faster manufacturing SMEs in the north-east (plant engineering).

- MTS, from Hungary, is facing foreign competition with a business organization typical of state-owned companies operating on local protected markets (system engineering).

- METZ, a German medium-sized firm delivering customized vehicles and vehicle equipment services, has to synchronize engineering, manufacturing and procurement activities in a typical one-of-akind manufacturing environment.
The pilot sites had an active role in all planning and implementation phases of the work prior to the demonstrations.

In the following sub-sections, the contents of each thematic area are discussed in more detail, to give an indication of the technical content, results and methodologies concerning each part of the work.

\subsection{Network architecture and software components}

\subsubsection{Approach and results}

Three different layers are identified: in the network logical architecture (see Fig. 5).

The Network Data Model is closely connected of the following three layers.

1. The Work Network Structure (WNS) constitutes the bottom layer of the system infrastructure, crossing horizontally functional silos at companies cooperating on large-scale projects. At this level, physical nodes are identified corresponding to autonomous organization units, and links are defined allowing integration of data and cooperation between nodes. The WNS gives visibility on the project network as a portfolio of assets that can be uniquely configured for each project, in terms of:

$\circ$ nodes specialization and capabilities, e.g. manufacturing, engineering, supply of components or services, management of project activities;

$\circ$ resources provided according to capabilities, e.g. products, manufacturing capacity, personnel, service manpower, equipment, with the corresponding cost and usage rates, along with knowledge and documents in various formats;

- availability of data by resource type, e.g. current and planned stocks, manufacturing calendars, employees schedule;

- status of allocated resources to specific projects, e.g. job orders' stages, advance of components supplies, involved employees' time sheets;

- links between nodes representing co-operation possibilities, e.g. materials or services supply, workload sub-contracting in specific activities, and fixing the terms and conditions for cooperation.

2. The Work Accountability Structure (WAS) constitutes the intermediate layer of the system 


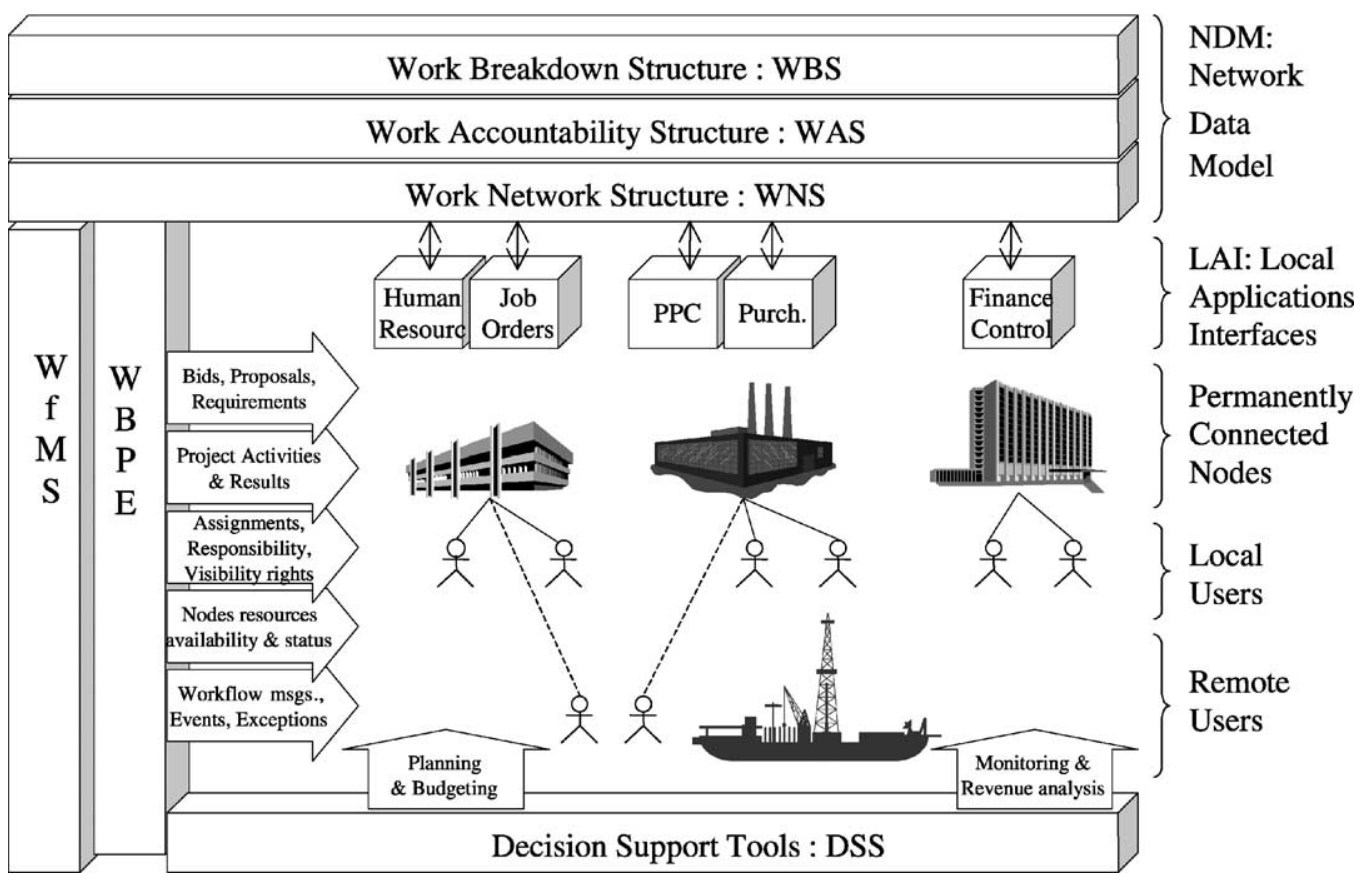

Fig. 5. The system IT architecture. WBPE: Web-based Project Environment; WfMS: Workflow Management System.

infrastructure, representing the temporary, multisite and multi-company organization created to carry out one or more projects. The WAS groups and links nodes and individuals are accessible through the WNS, to create a project-oriented structure specifically designed for the work to be carried out, including:

$\circ$ nodes and groups of nodes representing relevant project units, i.e. units occupying a specific position with reference to project planning, deployment and monitoring activities;

- roles played by project participants in each project unit, identified by competencies and skill levels, and explicitly assigned to personnel units identified in the WNS, including substitution alternatives and referees in the chain of responsibility;

- visibility rules to be applied for a specific unit or role, concerning access of project-related data and knowledge at the unit node(s) and access to other nodes' data through WNS links;

○ workflow model for the circulation of events and decision between units and actors at all levels in the WAS, depending on the assigned responsi- bilities (e.g. project budgeting) and co-operation foreseen between project units (e.g. joint revision of project plans).

3. The Work Breakdown Structure (WBS) corresponds to the topmost layer of the system infrastructure, and represents a network-wise enhancement of WBS implementations supported by traditional project planning tools. The WBS system describes the project network objectives and achievements, in terms of:

$\circ$ project activities, their type (e.g. manufacturing, services, management), detail level (e.g. simple activities, clusters), dependencies (e.g. milestones, time-related constraints), and alternatives; o input and output artifacts associated to each activity, i.e. products, components and services representing activities, requisites or measurable results for WNS nodes;

- resources requirements associated to each activity, mapped onto nodes' resource types (e.g. manpower categories, equipment, employees with proper knowledge and skills) for cost and availability evaluation, with indication of alternative resources; 
- planning details, including project milestones and activities' timing, allocation of nodes' resources and budget (cost and revenue) estimations by project unit, project planning status and revisions;

- Execution status for released or running activities, expressed by time- and cost-based indicators, artifacts produced, resources usage, referred to and compared with activities' planning data.

To support the outlined organization model, the system network is provided with innovative software tools, according to the IT architecture shown in Fig. 5.

1. Web-based Project Environment. The system architecture is centered on a Web-based Project Environment (WPE or WBPE) - a shared and integrated project management infrastructure that: 0 is based on web technologies, so as to be easily accessed by local users at network nodes (intranet access) and by remote users operating at decentralized project sites (Internet or extranet access);

- provides uniform access to data and functionalities from heterogeneous applications, including both our system components and local management and planning tools at any network node;

- allows data distribution and decentralized management, providing a unique entry point to information owned and maintained by different nodes in the real-world network structure;

- implements three levels of protection for nodes' data access: (i) for users of a node, visibility rights are assigned and verified at logon time; (ii) between nodes, data are protected by a decentralized visibility mechanism allowing each node to assign access rights on its data to other nodes, based on the data content and the relation with the accessing node; and (iii) at the technological level, each node hosting a system server will be protected against unauthorized access by a firewall.

2. Workflow Management System (WMS or WfMS). The WPE embeds process automation features provided by a commercial Workflow Management System, that:

$\circ$ allows process-based interaction between the responsible actors at the involved nodes, according to the roles, responsibilities and workflow model specified in the WAS (e.g. exchange of bids and requirements in the project negotiation phase, revision and approval cycle for project budgets);

o notifies relevant events and exceptions to the appropriate actors in the project network, depending on the event type and the action required (e.g. finding alternatives on resources shortage or equipment failure);

- can easily be accessed and configured to realize higher level functionalities, by integrating workflow management with system application data and Decision-support sub-systems.

3. Network Data Model (NDM). The system organization model requires a common data infrastructure provided by the Network Data Model-a distributed and decentralized database that:

- maintains an updated and consistent representation of the project network from each node point of view, i.e. including all the other nodes visible to and interacting with the current one;

- aggregates and normalizes information from nodes' local applications to realize the WNS model, allowing for both detail and synthesis views on physical nodes, their resources and activities status;

- allows consistent manipulation of WNS data into higher level WAS and WBS models, supporting both centralized and decentralized data management schemes (e.g. allocation of project packages to different nodes for joint planning and execution).

4. Local Applications Interfaces (LAI). Alignment between the new project network management and internal node activities will be provided by a set of Local Applications Interfaces, that:

- integrate data from nodes selected sources (manufacturing, human resources, finance and accounting) into the WNS model, making them accessible to the other nodes for decision-making processes, e.g.: node materials, manpower and equipment, their cost and availability, key personnel identification, job orders progress and activities accounting data;

○ wrap existing information system components, like ERP and PPC, to realize a set of system- and platform-independent objects (e.g. job order) with project-relevant properties and events (e.g. job order completion); particular attention will be paid to integration widespread ERP platforms, as SAP, Baan, Diapason, etc. 
- transfer network-level data and events to the node internal functions for local processing, e.g.: MRP planning of materials requirements associated to a project activity, issuing and managing purchase orders for network-planned services or components;

$\circ$ integrates with existing information retrieval and knowledge management facilities, to link consistently knowledge assets and skills at each node with activities and roles in the WBS and WAS network data structures.

5. Decision-support sub-systems. Two decision-support sub-systems are developed and deployed as add-ons to the rest of the software, respectively for: $\circ$ project planning and budgeting to support decision-makers in project ideation and bid preparation tasks, thanks to features like: (i) project scheduling based on WBS activities, their resources and materials requirements, time constraints, current and planned resources availability at each node, (ii) generation, quantitative evaluation and comparison of alternatives concerning, e.g.: selection of the most appropriate suppliers and sub-contractors, workload allocation on critical resources and equipment, and (iii) budget creation based on project schedule, WNS nodes costs, rules for budget allocation and expense calculation on WAS entities;

- project monitoring and revenue analysis to support project and site managers in evaluating activities' progress, identifying risk elements and launching contingency actions, thanks to features like: (i) real-time activities' monitoring, based on significant time, cost and performance indicators, derived from node data provided by local applications interfaces and compared with planned values, (ii) profitability analysis by project, projects groups and WAS units, based on cash-flow profiles and planned versus actual costs estimations, and (iii) "what if" scenarios evaluation, to simulate both deterministic and causal factors effects on the project plan.

\subsubsection{Software design and development and deployment}

To avoid replication of functionalities and waste of resources, our approach strongly relies on the integration with standard, and commercially available tools. The system is designed and implemented with common tools and principles [15-19], we applied as many standards as possible during each step of the system design, development, implementation and testing. The system uses a normal client/server architecture with a base client and a web server and application server and uses several free (with operating system) and some relatively cheap commercial tools. It runs on Intel-based personal computer platforms, with the following system requirements to ensure broad application possibilities:

a. Base Client.

- Internet Browser: Internet Explorer 6 with Microsoft XML Parser 3.0.

All client components are automatically downloaded as a $\mathrm{CAB}^{2}$ files and installed when the user accesses the system for the first time. Depending to the client machine configuration there are two possible scenarios:

- client machines with $\mathrm{ADO}^{3}$ and Visual Basic Runtime: no reboot required;

- client machines without ADO or VB Runtime: one or two reboots are needed, automatically managed by the set-up procedure;

- Operating system: Windows NT4 Workstation, Windows 2000 Professional.

- Office 2000 with Web Components (for MRADSS $^{4}$ ).

- SQL Server 2000 Analysis Services sp1 PivotTable Services PTSFull.exe (for MRADSS).

b. Web Server.

- Operating system: Windows 2000 Server with Service Pack 2 (minimum).

- Workflow Engine: Microsoft BizTalk Server 2000 version 1.0. Service Pack 1.

- Service components for SAIL ${ }^{5}$ VBGit and ScriptControl.

- SQL Server 2000 Analysis Services (for MRADSS).

\footnotetext{
${ }^{2} \mathrm{CAB}$ indicates Cabinet files (.cab), containing compressed files for programs installation via the Internet.

${ }^{3} \mathrm{ADO}$ is acronym of ActiveX Data Object, identifying the Microsoft high-level interface for accessing database objects.

${ }^{4}$ Monitoring and requirement analysis decision support system.

${ }^{5} \mathrm{SAIL}^{\mathrm{TM}}$ is Gruppo Formula standard framework for developing client/server applications.
} 
- MS Project 2000 (for PBDSS $^{6}$ ).

c. Application Server.

○ Operating system: Windows 2000 Server with Service Pack 2 (minimum).

- Service components for SAIL version 2.5.5b ${ }^{7}$ : VBGit and ScriptControl.

\subsection{Business cases analysis, implementation and evaluation}

The objective of the four business cases is to provide reference requirements, realistic applications on the field, and measures of the system benefits by applying our management model and tools and software on real-world projects carried out by pilot users. The system user companies have been selected to represent various types of engineering and service networks in different countries, thus providing a significant selection of business cases for requirements analysis and experimentation of the proposed approach (see right-hand side of Fig. 4: shipbuilding, engineering industry, plant repair and maintenance services and software project management).

We selected outward-focused modeling and benchmarking tools to comply with the system network organization model (e.g. standards oriented to supply-chain organizations [14]).

Recently, all four pilot cases are making the experimentation with the software tools and means and the results seem to be remarkable, however some more months are necessary to the appropriate evaluation.

To assist evaluation:

- the metrics defined and measured in preliminary business cases analysis will be measured again by the end of the experiments at the selected industrial sites;

- a comparison will be made between the initial values of the performance metrics and the final ones, and conclusions will be derived from that comparison.

This way, the assessment of the benefits will be quite straightforward to show that the new tools and working software are useful at real users' sites of four

\footnotetext{
${ }^{6}$ Planning and budgeting decision support system.

${ }^{7}$ SAIL $^{\mathrm{TM}}$ is Gruppo Formula standard framework for developing client/server applications.
}

different European countries. Each of the four cases, depending on the scope and industrial sector, needed a specific configuration of system modules to be implemented in order to carry out the experimentation work. The installations proved that the solutions are general enough to be easily implemented.

\section{Conclusions}

The implementation of our logistics flow management/supply-chain approach represents a significant step forward on state-of-the-art logistics management techniques for the end-users. On the one side, in traditional enterprise practice the focus is on bilateral supply relations with each individual customer and supplier, with scant and informal co-operation possibilities and no supply-chain visibility. On the other side, multi-site planning extensions offered by major ERP and SCP vendors are still based on a centralized approach, lacking on-line integration and synchronization with the other network actors. In this scenario, we provide considerable benefits in terms of improved network visibility, better coordination and real-time control of materials flows.

Feasibility of the above improvements, along with the costs and time required for achieving them, are assessed through experimentation of the software system on selected user firms. The validation phase was successfully finished on four pilot cases in different industrial sectors: machine-tool industry, equipment production, textile industry and naval industry.

Experimentation was done on the basic flow management components, supporting network modeling, data-integration and workflow, in parallel with design and development of the decision-support components. Now the software is finished and it is commercially available.

The web-based management software provides a planning and management infrastructure for complex distributed organizations working on large-scale engineering projects, characterized by huge investments in both materials and human resources and by concurrent, disparate activities-manufacturing, design and services as well. The first experiments are running successfully at all the four different pilot sites and will prove all advantages detailed in this paper. 


\section{Acknowledgements}

The authors would like to acknowledge the support of the European Union that provided the funds for the Research Projects FLUENT (Esprit IiM-1998-29088) and WHALES (Esprit IST-1999-12538) the key results of which are presented in this paper. We are also thankful for all Italian, Portuguese, Greek, German and Hungarian academic and industrial partners for their co-operation.

\section{References}

[1] FLUENT, Esprit IiM-1998-29088: Flow-oriented Logistics Upgrade for Enterprise Networks, EU project documentations.

[2] WHALES, Esprit IST-1999-12538: Web-linking Heterogeneous Applications for Large-scale Engineering and Services, EU project documentations.

[3] EP 20377 OPAL, Esprit 20377, OPAL: Integrated Information and Process Management in Manufacturing Engineering.

[4] EP 20408 VEGA, Esprit 20408, VEGA: Virtual Enterprise Using Groupware Tools and Distributed Architecture.

[5] IMS GLOBEMAN21, Globeman21 is an IMS (Intelligent Manufacturing Systems) project involving European, US and Japanese partners to develop the framework of globally networked manufacturing in the 21st century.

[6] EP 26854 VIVE, Esprit 26854, VIVE: Virtual Vertical Enterprise.

[7] M. Hopf, Holonic manufacturing systems (HMS) - the basic concept and a report of IMS Test Case 5, in: J.H.K. Knudsen, et al. (Eds.), Sharing CIME Solutions, Ios Press, 1994.

[8] A. Rolstadas, Beyond Year 2000-Production Management in the Virtual Company, IFIP Transactions B-19: Production Management Methods, North Holland, 1994.

[9] B.E. Hirsch, et al., Decentralized and collaborative production management in distributed manufacturing environments, in: J.H.K. Knudsen, et al. (Eds.), Sharing CIME Solutions, Ios Press, 1995.

[10] F. Bonfatti, P.D. Monari, P. Paganelli, Coordination Functions in a SME Network, in: Proceedings of BASYS '96 International Conference on Balanced Automation Systems, vol. II, Chapman \& Hall, Lisbon, 1996.

[11] Price Waterhouse ${ }^{\mathscr{C}}$, Supply chain management practice, in: Supply Chain Planning for Global Supply Chain Management, 1997.

[12] V. Mirchandani, J. Block, Supply Chain Management and the Back Office, Gartner Group ${ }^{C}$ Strategic Analysis Report, 1996.

[13] EP 20876 ELSEWISE, Esprit 20876: European LSE Wide Integration Support Effort.

[14] SCOR Model, The Supply Chain Operations Referencemodel (SCOR) has been developed and endorsed by the Supply-Chain Council (SCC), http://www.supply-chain.org/.

[15] I. Mezgár, Communication Infrastructures for Virtual Enterprises, position paper at the panel session on Virtual
Enterprising - the way to Global Manufacturing, in: R. Traunmuller, E. Csuhaj-Varju (Eds.), Proceedings of the IFIP World Congress, Teleco-operation, Vienna, Austria and Budapest, Hungary, 31 August-4 September, pp. 432-434.

[16] COM, Sessions R., COM and DCOM-Microsoft Vision of Distributed Objects, Wiley Computer Publishing, 1998, ISBN 0-471-19381-X, http://www.microsoft.com/.

[17] CORBA Standard, Object Management Group, CORBA/IIOP 2.2 specification, 1998, ${ }^{\circ}$ Object Management Group, http:// www.omg.org/.

[18] UML, Rational Corporation, UML Notation Guide, Version 1.1, 1997, ${ }^{\mathbb{C}}$ Rational Corporation and UML Partners, http:// www.rational.com/.

[19] I. Jacobson, M. Christerson, P. Jonsson, G. Overgaard, Object Oriented Software Engineering: A Use Case Driven Approach, OOSE Methodology, Addison Wesley, 1993, ISBN 0201-54435-0.

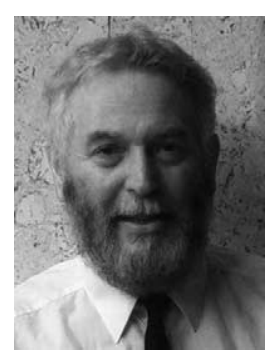

George L. Kovács got his masters and $\mathrm{PhD}$ from the, Faculty of Electrical Engineering, Technical University of Budapest in 1966 and 1976, respectively. He is a professor at the same university since 1995 (Faculty of Mechanical Engineering). In 1997 he got the doctor of the academy degree from the Hungarian Academy of Sciences (HAS). He is with the Computer and Automation Research Institute of HAS since 1966, recently he was appointed as the head of the CIM Research Laboratory. He is also a visiting researcher in the US, Soviet Union, West Germany, Mexico and at University of Trento in Italy. He has authored more than 250 scientific publications. Kovács is a member of several Hungarian and international scientific organizations, including IEEE, IFAC and IFIP. His scientific interest is intelligent manufacturing. He is a project manager of several Hungarian and international R\&D projects.

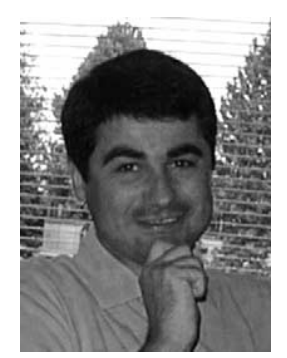

Paolo Paganelli graduated in electronic engineering at the University of Bologna in 1991. From 1992 to 1994 worked as research assistant at the University of Modena, involved in design and development of innovative tools to support advanced production planning. From 1994 to 1997, he worked at Democenter, regional center for diffusion of CIM technologies, at the R\&D department. In 1997, he joined Gruppo Formula, the major ERP software producer in Italy, as senior analyst involved in the design of Formula's next generation ERP software, and since year 2000 he is Formula's Product Manager for SupplyChain Management solutions. He has been the project manager of IST projects FLUENT and WHALES. His main research interests are in product and process data modeling, supply-chain management and virtual/extended enterprise. He has published around 30 papers (at international conferences, in scientific journals and as technical reports. 